\title{
Generating function for tensor network diagrammatic summation
}

\author{
Wei-Lin Tu $\odot,{ }^{1,2}$ Huan-Kuang Wu $\odot,{ }^{3}$ Norbert Schuch, ${ }^{4,5,6,7}$ Naoki Kawashima, ${ }^{1}$ and Ji-Yao Chen $\odot^{4,5, *}$ \\ ${ }^{1}$ Institute for Solid State Physics, University of Tokyo, Kashiwa, Chiba 277-8581, Japan \\ ${ }^{2}$ Division of Display and Semiconductor Physics, Korea University, Sejong 30019, Korea \\ ${ }^{3}$ Department of Physics, Condensed Matter Theory Center and Joint Quantum Institute, University of Maryland, \\ College Park, Maryland 20742, USA \\ ${ }^{4}$ Max-Planck-Institut für Quantenoptik, Hans-Kopfermann-Straße 1, 85748 Garching, Germany \\ ${ }^{5}$ Munich Center for Quantum Science and Technology, Schellingstraße 4, 80799 München, Germany \\ ${ }^{6}$ Faculty of Physics, University of Vienna, Boltzmanngasse 5, 1090 Wien, Austria \\ ${ }^{7}$ Faculty of Mathematics, University of Vienna, Oskar-Morgenstern-Platz 1, 1090 Wien, Austria
}

(Received 27 January 2021; revised 6 May 2021; accepted 13 May 2021; published 28 May 2021)

\begin{abstract}
The understanding of complex quantum many-body systems has been vastly boosted by tensor network (TN) methods. Among others, excitation spectrum and long-range interacting systems can be studied using TNs, where one however confronts the intricate summation over an extensive number of tensor diagrams. Here, we introduce a set of generating functions, which encode the diagrammatic summations as leading-order series expansion coefficients. Combined with automatic differentiation, the generating function allows us to solve the problem of TN diagrammatic summation. We illustrate this scheme by computing variational excited states and the dynamical structure factor of a quantum spin chain, and further investigating entanglement properties of excited states. Extensions to infinite-size systems and higher dimension are outlined.
\end{abstract}

DOI: 10.1103/PhysRevB.103.205155

\section{INTRODUCTION}

The study of quantum many-body systems using tensor networks (TNs) has witnessed great success in the last three decades [1-3]. Originally, TN methods were developed to efficiently capture ground-state properties of many-body lattice models with short-range interaction [4-7]. Later on, numerous progress has been made in various directions, including determining low-energy excited states [8], exploring dynamical and finite temperature properties [9], and finding valuable applications in long-range interacting systems $[10,11]$. These developments not only deepen our theoretical understanding of many-body systems [12] but also bridge TN methods to real experiments [13].

New developments also bring challenges, however. Both quasiparticle excited states [5,14-16] and global observables contain contributions with a sizable number of tensor diagrams, due to the fact that quasiparticle or local operators of global observables can be on an arbitrary patch of the lattice. Except in a few cases where efficient summation techniques have been proposed [17-20], most notably, the matrix product operator (MPO) representation of global observables [19],

\section{*Ji-Yao.Chen@mpq.mpg.de}

Published by the American Physical Society under the terms of the Creative Commons Attribution 4.0 International license. Further distribution of this work must maintain attribution to the author(s) and the published article's title, journal citation, and DOI. Open access publication funded by the Max Planck Society. extensive and costly tensor diagram manipulation seems to be unavoidable and becomes the bottleneck in modern TN applications. Thus an efficient and universal approach for TN diagrammatic summation is highly called for.

Another domain where diagrammatic summations frequently appear is the perturbation theory of interacting quantum fields $[21,22]$. There, for correlation functions containing summations of Feynman diagrams, one can introduce a source field and formally represent correlation functions as derivatives of the perturbed partition function, known as the generating functional method [21]. Given the close relation between the trio of TN methods, many-body systems, and quantum field theory (QFT), and the pictorial similarity of the tensor diagram and Feynman diagram, it is tempting to look for a generating function formalism in TNs, where certain derivatives can compactly represent the summations of TNs. This is plausible, as partition functions of classical statistical models are known to be representable as TNs [23].

In this paper, inspired by the generating functional method in QFT, we propose a set of generating functions for TNs, which encode TN diagrammatic summations as leading-order expansion coefficients. It then requires taking derivatives of the generating functions, which can be accomplished with automatic differentiation (AD) [24-26]. To illustrate the scheme, we investigate the low-lying spectrum of a quantum spin chain with periodic uniform matrix product state (MPS) and the excitation ansatz [14], and subsequently study entanglement properties of excited states which, to our knowledge, were rarely studied due to the overwhelmingly large number of tensor diagrams involved. 


\section{PERIODIC UNIFORM MPS AND EXCITATIONS}

Let us consider a translationally invariant quantum spin chain with $N$ sites. For simplicity, we assume that the ground state is unique and can be approximated by a periodic uniform MPS with the form:

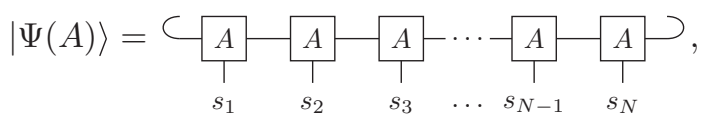

where the same rank-3 tensor $A$ with dimension $d \times D \times D$ is repeated on every site, and the left boundary is contracted with the right boundary. Here, $s_{i}=1, \ldots, d$ represents the basis of $d$-dimensional local Hilbert space, while $D$ is the virtual bond dimension, controlling the accuracy of the MPS ansatz. We further denote the one-site translation operator as $\hat{T}$ with $\hat{T}\left|s_{1}, s_{2}, \ldots, s_{N}\right\rangle=\left|s_{N}, s_{1}, \ldots, s_{N-1}\right\rangle$, which satisfies $\hat{T}^{N}=1$. By construction, $|\Psi(A)\rangle$ is translationally invariant with momentum $k=0$, i.e., $\hat{T}|\Psi(A)\rangle=|\Psi(A)\rangle$. For a given model, the ground-state tensor $A$ can be optimized using the conjugate gradient method, with gradient obtained from AD of the computation graph for energy [26,27].

With ground-state tensor at hand, excited states can be constructed using the single mode approximation [28], which correspond to one-particle excitation and work well for a broad range of models [29-31]. In full generality, one can perturb the ground state by replacing one site tensor $A$ with a new tensor $B$ which is yet to be determined, and then build up a Bloch state using the translation operator [5,14], taking the form:

$$
\left|\Phi_{k}(B)\right\rangle=\sum_{j=0}^{N-1} \mathrm{e}^{-i k j} \hat{T}^{j} \underbrace{B}_{s_{1}} \underbrace{A}_{s_{2}} \ldots s_{s_{N}}^{A} \cdots,
$$

where tensor $B$ contains variational parameters for the excited state. $\left|\Phi_{k}(B)\right\rangle$ then is an eigenstate of the translation operator with eigenvalue $\mathrm{e}^{i k}$, where momentum $k=$ $2 \pi m / N, m=0,1, \ldots, N-1$. Due to momentum superposition, a summation of $N$ different tensor diagrams appears in Eq. (2), which will be our main focus. Since $\left|\Phi_{k}(B)\right\rangle$ depends on tensor $B$ linearly, variationally optimizing $B$ boils down to a generalized eigenvalue problem: $\mathbf{H}_{\mu \nu} \mathbf{B}^{v}=$ $E \mathbf{N}_{\mu \nu} \mathbf{B}^{\nu}$, where $E$ is the generalized eigenvalue and $\mathbf{H}(\mathbf{N})$ is the effective Hamiltonian (norm) matrix in the variational space, with $\mathbf{H}_{\mu \nu}=\frac{\partial^{2}}{\partial \overline{\mathbf{B}}^{\mu} \partial \mathbf{B}^{v}}\left\langle\Phi_{k}(B)|\hat{H}| \Phi_{k}(B)\right\rangle, \mathbf{N}_{\mu \nu}=$ $\frac{\partial^{2}}{\partial \overline{\mathbf{B}}^{\mu} \partial \mathbf{B}^{v}}\left\langle\Phi_{k}(B) \mid \Phi_{k}(B)\right\rangle$. Here, $\bar{B}$ is the complex conjugate of $B$, whose component after vectorization is denoted as $\mathbf{B}^{v}$. Since momentum is a good quantum number, we have suppressed the dependence of $\mathbf{H}, \mathbf{N}, E$, and $\mathbf{B}$ on momentum $k$. Solving the generalized eigenvalue equation in each momentum sector, one recovers the low-energy spectrum [32].

To construct $\mathbf{H}$ and $\mathbf{N}$, one needs to sum over $N$ different tensor diagrams for each, with MPO representation of the Hamiltonian $\hat{H}$. These extensive tensor diagram summations are the main obstacles of computing the excitation ansatz, rendering manipulating excited states unfavorable. Below we introduce our formalism based on simple yet powerful generating functions with the following strategy: To compute $\mathbf{H}$ or $\mathbf{N}$, we will first construct a suitable generating function and then use AD to compute the derivative [26], which will reproduce $\mathbf{H}$ or $\mathbf{N}$ and is much simpler than directly summing all diagrams. In this way, we will get rid of all the tensor diagram summations, making it possible to investigate detailed properties of excited states. Note that, unlike generating functionals in QFT, whose closed-form expressions are rare, the TN generating functions and their derivatives can be computed numerically exact with AD. We find that, depending on the origins of diagrammatic summation, the generating functions can be divided into two classes, one for TN state and the other for TN operators, which we introduce separately.

\section{GENERATING FUNCTION FOR STATE}

As shown in Eq. (2), the extensive tensor diagrams only differ by the location of tensor $B$ and a position-dependent phase factor. It is insightful to make the following observation: For a given tensor $B$, the excitation ansatz equation (2) can be expressed as $\left|\Phi_{k}(B)\right\rangle=\left.\frac{\partial}{\partial \lambda}\left|G_{\Phi}(\lambda)\right\rangle\right|_{\lambda=0}$, with

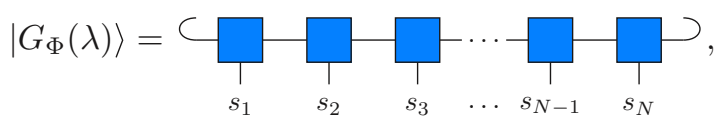

where the tensor on the $j$ th site in $\left|G_{\Phi}(\lambda)\right\rangle$ is given by $A_{j}(\lambda)=$ $A+\lambda \mathrm{e}^{-i k(j-1)} B, \lambda \in \mathbb{R}$, represented by blue squares. Here, to simplify the notation, we have suppressed the dependence of $\left|G_{\Phi}(\lambda)\right\rangle$ on tensor $A, B$ and momentum $k$, keeping only $\lambda$ dependence explicitly. Expanding $\left|G_{\Phi}(\lambda)\right\rangle$ into a power series of $\lambda$, we find that the ground (excited) state $|\Psi(A)\rangle\left(\left|\Phi_{k}(B)\right\rangle\right)$ is contained in the zeroth-order (first-order) term, both of which lie in the tangent space of the MPS manifold, while higher-order terms are outside of the tangent space due to nonlinearity in tensor $B$ [33]. Thus we can eliminate the tensor diagram summation in $\left|\Phi_{k}(B)\right\rangle$ by computing the first-order derivative of $\left|G_{\Phi}(\lambda)\right\rangle$. Interestingly, Eq. (3) bears a similarity with the generating functional in QFT, where the parameter $\lambda$ plays the role of source field in the latter. Note that, although $|\Psi(A)\rangle$ and $\left|\Phi_{k}(B)\right\rangle$ are translationally invariant, the generating function $\left|G_{\Phi}(\lambda)\right\rangle$ is in general not invariant under one-site translation, except at momentum $k=0$.

With $\left|G_{\Phi}(\lambda)\right\rangle$, the norm square of the excited state can be expressed as $\||\Phi\rangle \|^{2} \equiv\left\langle\Phi_{k}(B) \mid \Phi_{k}(B)\right\rangle=$ $\left.\frac{\partial^{2}}{\partial \lambda^{\prime} \partial \lambda}\left\langle G_{\Phi}\left(\lambda^{\prime}\right) \mid G_{\Phi}(\lambda)\right\rangle\right|_{\lambda^{\prime}=\lambda=0}$. Using translation invariance of $\left|\Phi_{k}(B)\right\rangle$, we can lower the order of the derivative with the following generating function for the excited-state norm:

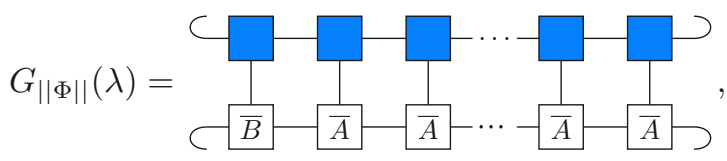

with which the norm square can be obtained as $\||\Phi\rangle \|^{2}=$ $\left.N \frac{\partial}{\partial \lambda} G_{|| \Phi||}(\lambda)\right|_{\lambda=0}$. Here, the local tensor on site $j$ of the ket layer is $A_{j}(\lambda)$, the same as appearing in Eq. (3).

Before proceeding further, let us discuss how to use generating functions in practice. Taking Eq. (4) as an example, we first compute $G_{\| \Phi||}(\lambda=0)$ by contracting the network in a conventional manner with computational complexity $O\left(D^{5}\right)$, followed by a backpropagation using $\mathrm{AD}$, with which the first-order derivative at $\lambda=0$ is obtained automatically, hence 
the norm square [26]. Since the computational complexity of $\mathrm{AD}$ grows with the order of the derivative, it is advisable to utilize a generating function with which a low-order derivative suffices.

With Eq. (4), it is straightforward to find the generating function for the norm matrix $\mathbf{N}$ :

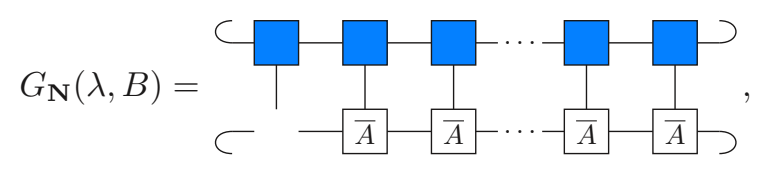

where we simply omit tensor $\bar{B}$ in the bra layer of $G_{\| \Phi||}(\lambda)$. From Eq. (5), the norm matrix $\mathbf{N}$ can be obtained as $\mathbf{N}=$ $\left.N \frac{\partial}{\partial B} G_{\mathbf{N}}(\lambda, B)\right|_{\lambda=1, B=0}$. Unlike the scalar $G_{\| \Phi||}(\lambda)$ where the derivative is taken with respect to $\lambda$ at $\lambda=0, G_{\mathbf{N}}(\lambda, B)$ is a vector, and the derivative is taken with respect to $B$ at $\lambda=1, B=0$, which can also be computed with AD [34].

\section{GENERATING FUNCTION FOR OPERATOR}

Another type of TN diagrammatic summation originates from global observables. For example, when computing the structure factor, one needs to take into account correlations of the local operator at all distances. Moreover, when applying TN methods to long-range interacting models which appear naturally in Rydberg quantum gas and quantum chemistry systems, efficient and faithful encoding of the long-range interaction is one of the main issues [35]. Although approaches based on the MPO and projected entangled-pair operator (PEPO) have been proposed, the bond dimension can be quite large, making them less appealing [36-39]. Here, we provide generating functions for global observables, without involving the MPO whenever possible.

Let us first consider the spin structure factor. Here, the summations arise from the Fourier transform of the onsite spin operator $\hat{S}_{j}^{\alpha}$ (index $\alpha$ is in operator space): $\hat{S}_{k}^{\alpha}=$ $\frac{1}{\sqrt{N}} \sum_{j=1}^{N} \mathrm{e}^{-i k j} \hat{S}_{j}^{\alpha}$. A generating function can be introduced as follows:

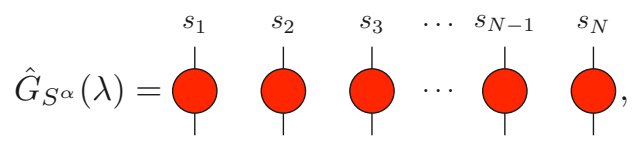

which is a tensor product of on-site operators, with $\hat{S}_{j}^{\alpha}(\lambda)=$ $\mathbb{I}+\lambda \mathrm{e}^{-i k j} \hat{S}_{j}^{\alpha}, \lambda \in \mathbb{R}$, acting on the $j$ th site. One can then find $\hat{S}_{k}^{\alpha}=\left.\frac{1}{\sqrt{N}} \frac{d}{d \lambda} \hat{G}_{S^{\alpha}}(\lambda)\right|_{\lambda=0}$. Taking two layers of Eq. (6) with independent parameters $\lambda, \lambda^{\prime}$, followed by a second-order derivative with respect to $\lambda$ and $\lambda^{\prime}$, one arrives at the static structure factor. Higher-order moments of the local operator can be similarly obtained. Note that, with translation symmetry, one can combine $\hat{G}_{S^{\alpha}}(\lambda)$ and the local spin operator to reduce the order of the derivative.

Interestingly, by removing the $\lambda$ dependence in the first operator and replacing the phase factor in the others with the corresponding distance-dependent coefficient, Eq. (6) can be used to efficiently encode long-range power-law decaying interactions. Moreover, this approach can be easily generalized to higher dimension, where the PEPO technique for long-range interactions is imperfect [37]. A simple counting suggests that the number of terms for expectation value of long-range interaction is $N^{2}$, while with generating function equation (6), it is only of order $N$, where each term differs by the position of the first operator. Exploiting translation symmetry, the latter case can be further reduced to a single term. Without translation symmetry, a combination of Eq. (6) and the MPO technique is possible, which may lower the bond dimension of the MPO.

While Eq. (6) relies on the fact that $\hat{S}^{\alpha}$ is an on-site operator, the scheme can be generalized to the case where the local operator has support on more than one site, e.g., the local Hamiltonian operator. Similar to Trotter gates, we introduce the following generating function for the Hamiltonian operator under periodic boundary condition $\hat{H}=\sum_{j=1}^{N} \hat{h}_{j, j+1}$ (assuming that $N$ is even and nearest-neighbor interaction):

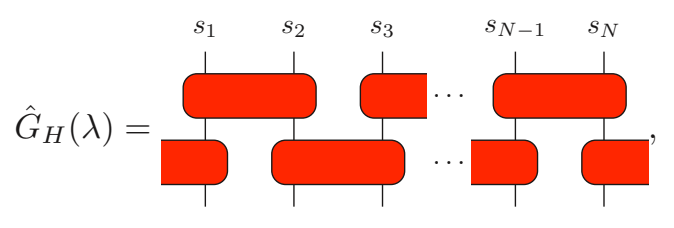

where the operator acting on the $(j, j+1)$ bond is given by $\hat{h}_{j, j+1}(\lambda)=\mathbb{I}+\lambda \hat{h}_{j, j+1}, \quad \lambda \in \mathbb{R}$, represented by a red rectangle. It is then straightforward to verify $\hat{H}=$ $\left.\frac{d}{d \lambda} \hat{G}_{H}(\lambda)\right|_{\lambda=0}$. For the effective Hamiltonian in the generalized eigenvalue equation, one could take the generating function for norm matrix equation (5) and insert a MPO representation of the Hamiltonian, or the generating function equation (7) with an additional derivative. The latter could be advantageous if the MPO has a relatively large bond dimension [40]. Removing the boundary term, $\hat{G}_{H}(\lambda)$ can also be applied for systems with an open boundary. Furthermore, taking two layers of $\hat{G}_{H}(\lambda)$, one can efficiently compute the energy variance.

A close comparison between generating functions for TN state, operator, and partition function in QFT suggests that, for excited state, it is constructed by introducing a source field to the ground-state tensor, while for operators, the identity operator is taken as the reference. This formalism also reminds us of Lie algebra, whose generators can be obtained by expanding group elements around the identity. In that case, the generating function has an exponential form. Indeed, generating functions for moments and cumulants have been introduced in Ref. [41], which took exponential forms with possible Trotter error and a finite difference method was then employed. In contrast, our approach does not have Trotter error, or finite difference error, and the generating functions for the state and operator can be unified in a simple way.

\section{NUMERICAL RESULTS}

\section{A. Variational excited states and dynamical structure factor}

We now present the numerical results using generating functions. The model we use for a benchmark is the spin-1 Heisenberg chain with Hamiltonian $\hat{H}=J \sum_{j=1}^{N} \mathbf{S}_{j} \cdot \mathbf{S}_{j+1}$, where $\mathbf{S}_{j}=\left(\hat{S}_{j}^{x}, \hat{S}_{j}^{y}, \hat{S}_{j}^{z}\right)$ are spin-1 operators on site $j$ and $J=1$ is taken as the energy unit. We use the periodic 


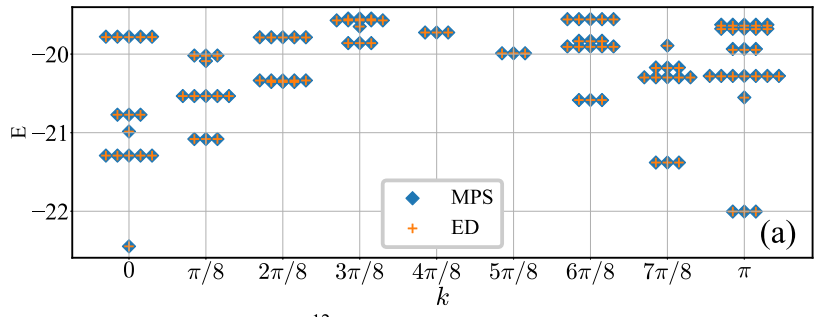

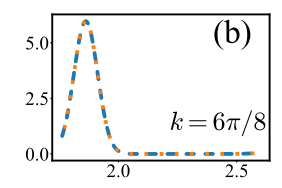

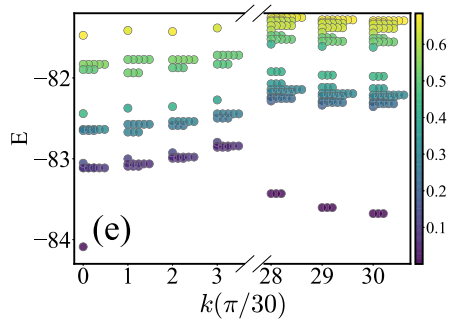

FIG. 1. Spectral properties of the spin-1 Heisenberg chain. (a) shows a comparison of the $D=24$ variational MPS calculation with ED for system size $N=16$. Further comparison of DSF at $k=3 \pi / 4,7 \pi / 8, \pi$ is shown in (b)-(d). In (e), we show the lowenergy spectrum with size $N=60$, obtained with $D=30$ MPS, where color represents the energy variance. Based on the spectrum in (e), DSF is computed and shown in (f) with a clear peak around $(k, \omega)=(\pi, 0.4)$.

uniform MPS to approximate the ground state and compute the low-energy spectrum with the excitation ansatz, where generating functions introduced above are used. For small system size $(N=16)$, we compare the variational energy spectrum with that obtained from exact diagonalization (ED). As shown in Fig. 1(a), with bond dimension $D=24$, the variational result agrees remarkably well with $\mathrm{ED}$, with maximal relative deviation $6 \times 10^{-4}$ for high energy levels, and the level degeneracy is also recovered to a high precision. Moreover, although the low-energy excitations around $k=0$ are two-magnon continuum [42,43], it is nevertheless reproduced well by the one-particle excitation ansatz.

With excited states available, dynamical properties of the system can be investigated in a straightforward manner by constraining the total Hilbert space to subspace spanned by variational ground and excited states [44]. One of the key observables is the dynamical spin structure factor (DSF), which reveals properties of quasiparticles and is accessible in neutron scattering experiments. The DSF is defined as $S^{\alpha}(k, \omega)=\sum_{n}\left|M_{k}^{\alpha}\right|^{2} \delta\left(\omega-E_{n}^{k}+E_{0}\right)$, with $M_{k}^{\alpha}=$ $\left|\left\langle\Phi_{k}\left(B_{n}\right)\left|\hat{S}_{k}^{\alpha}\right| \Psi(A)\right\rangle\right|$ and $E_{0}\left(E_{n}^{k}\right)$ being the energy of ground state $|\Psi(A)\rangle$ ( $n$th excited state $\left|\Phi_{k}\left(B_{n}\right)\right\rangle$ with momentum $k$ ). The delta function is replaced by a normalized Gaussian with broadening width $\sigma=0.05$, and we further take $\hat{S}^{z}$ as the operator in the DSF. The DSF can be efficiently computed with generating function equations (3) and (6), and the results for $k=3 \pi / 4,7 \pi / 8, \pi$ are shown in Figs. 1(b)-1(d) with system size $N=16$. Comparing with $\mathrm{ED}$, the line shapes are reproduced well by excitation ansatz.

For larger system size $(N=60)$, we quantify the quality of the variational result with the energy variance $\operatorname{Var}(E)=$ $\left\langle\hat{H}^{2}\right\rangle-\langle\hat{H}\rangle^{2}$, computed using generating functions [see Eqs. (4) and (7)]. Here, the bond dimension is $D=30$. As shown in Fig. 1(e), the energy variance of the variational ground state and one-magnon excited states remains small (on the order of $1 \times 10^{-3}$ ), considering the large system size, while the variational multimagnon excitations appear to have larger energy variances. The Haldane gap can be read off as $\Delta=0.4105$, in agreement with Refs. [29,43]. Further evaluating DSF using excited states [shown in Fig. 1(f)], we find a strong peak appearing at $(k, \omega)=(\pi, \Delta)$, where the first excited state is located. This confirms that the elementary excitation is magnon with momentum $k=\pi$, in agreement with the variational spectrum where the first excited state is a triplet at $k=\pi$. Vanishing DSF around $k=0$ is also consistent with excitations being two-magnon continuum in that region [43].

\section{B. Rényi entropy of excited states}

Apart from the variational energy spectrum and DSF, the generating functions also allow us to further study properties of excited states in great detail. Here, we use them to investigate entanglement properties of excited states, e.g., Rényi entropy, which has received considerable interest in recent studies [45-48]. It is well known that for one-dimensional gapped systems, the entanglement entropy of the ground state saturates with increasing subsystem size [49]. However, much less is known for excited states. Traditionally, computing Rényi entropy for excited states requires multiple summations and thus is hard to achieve. Here, we explore this question using generating functions without any summations.

For a normalized excited state $|\Phi\rangle$ with bipartition of the system $L$ and $\bar{L}$, the reduced density matrix (RDM) of subsystem $L$ (with size $l$ ) is given by $\rho_{L}=\operatorname{Tr}_{L}|\Phi\rangle\langle\Phi|$, and the Rényi entropy is then defined as $S^{(n)}=\frac{1}{1-n} \ln \operatorname{Tr}_{L} \rho_{L}^{n}$. Here, we will focus on the $n=2$ case, which can be computed by taking two copies of $\left|G_{\Phi}(\lambda)\right\rangle$ and $\left\langle G_{\Phi}(\lambda)\right|$, each with an independent parameter $\lambda$. Through AD of a single diagram, a fourth-order derivative (one for each layer) at the zero point gives directly the Rényi entropy.

The Rényi entropy with $n=2$ for the spin-1 Heisenberg model is shown in Fig. 2, using excited states in Fig. 1(e). The saturation of ground-state Rényi entropy with increasing $l$ is evident in the inset of Fig. 2, as expected. However, for excited states, we find that generically the Rényi entropy does not saturate with increasing $l$. To further quantify the effect of quasiparticles in excited states, we consider the ratio between excited- and ground-state traces: $F^{(2)}=\frac{\operatorname{Tr} \rho_{\Phi}^{2}}{\operatorname{Tr} \rho_{\psi}^{2}}$ [45], where $\rho_{\Phi}\left(\rho_{\Psi}\right)$ is the RDM of the excited (ground) state with subsystem size $l$. Theoretical studies have shown that, under the assumption of large momentum and energy gap, $F^{(2)}$ takes a universal form [47]: $F^{(2)}=x^{2}+(1-x)^{2}$, with $x \equiv l / N$. Indeed, the $k=\pi / 2$ result agrees with the theoretical prediction, although our model is neither integrable nor free. However, clear deviation is also observed for other momenta, which could be ascribed to the small energy gap or momentum and deserves further study. 


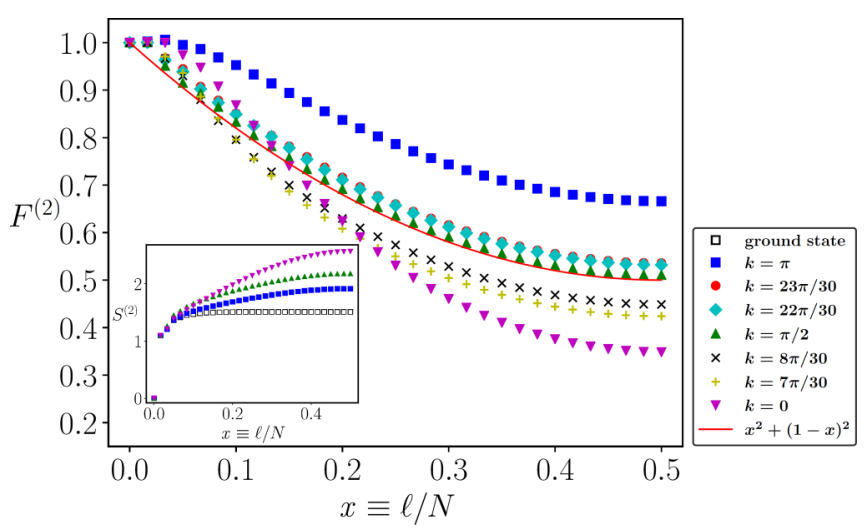

FIG. 2. Rényi entropy of excited states for the spin-1 Heisenberg model. In the main panel, different symbols represent the quantity $F^{(2)}$ of the first excited state at various $k$ with subsystem size $x \equiv$ $l / N$. The red line shows the theoretical prediction. Typical Rényi entropy with subsystem size is shown in the inset.

\section{DISCUSSION}

Above we have shown that the generating functions can be used to compute the one-particle excitation spectrum of a quantum spin chain with finite length. The results are encouraging, as the low-energy content is fully captured by MPS excitations, finding direct applications to investigate edge properties of two-dimensional (2D) systems [3]. The generating functions can also be adapted for tangent-space based excitation ansatz without translation symmetry [50]. There are several directions for further extension. To go beyond the one-particle case, one option would be modifying the ground-state tensor $A$ with $A+\sum_{i} \lambda_{i} B_{i}$, similar to introducing several source fields in the partition function of QFT. For infinite-size systems, one can combine generating functions with fixed-point methods, which will enable computing the excitation spectrum of 2D systems with projected entangled-pair states, including anyonic excitations in topological phases of matter [3].

\section{CONCLUSION AND OUTLOOK}

In this paper, we have introduced a set of generating functions for both TN states and TN operators, thereby eliminating extensive diagrammatic summations in modern applications of TN methods. Using generating functions, we have shown that the excitation spectrum of a quantum spin chain can be computed efficiently and accurately with the periodic uniform MPS, and the procedure is formally the same as ground-state search using TNs. Moreover, the generating functions allow us to investigate the dynamical structure factor of the system and the entanglement property of excited states in a convenient way, the latter of which is beyond the capability of traditional methods. We envision the generating functions introduced here will be powerful in the next generation of tensor network algorithms and applications.

Note added. Recently, we became aware of an independent work by Ponsioen and Corboz [51].

\section{ACKNOWLEDGMENTS}

We are thankful for useful conversations with Hyun-Yong Lee, Fa-Hui Lin, Cosimo C. Rusconi, Hong-Hao Tu, and Jiaju Zhang. Part of the numerical calculation was performed at the Supercomputer Center, ISSP, University of Tokyo. W.L.T. and N.K. are supported by JSPS KAKENHI Grant No. JP19H01809. H.-K.W. is supported by JQI-NSF-PFC (NSF Grant No. PHY-1607611). J.-Y.C. and N.S. acknowledge support by the European Union's Horizon 2020 programme through the ERC Starting Grant WASCOSYS (Grant No. 636201) and the ERC Consolidator Grant SEQUAM (Grant No. 863476), and from the Deutsche Forschungsgemeinschaft (DFG, German Research Foundation) under Germany's Excellence Strategy (Grant No. EXC-2111-390814868).
[1] U. Schollwöck, The density-matrix renormalization group in the age of matrix product states, Ann. Phys. 326, 96 (2011).

[2] R. Orús, Tensor networks for complex quantum systems, Nat. Rev. Phys. 1, 538 (2019).

[3] I. Cirac, D. Perez-Garcia, N. Schuch, and F. Verstraete, Matrix product states and projected entangled pair states: Concepts, symmetries, and theorems, arXiv:2011.12127.

[4] S. R. White, Density Matrix Formulation for Quantum Renormalization Groups, Phys. Rev. Lett. 69, 2863 (1992).

[5] S. Östlund and S. Rommer, Thermodynamic Limit of Density Matrix Renormalization, Phys. Rev. Lett. 75, 3537 (1995).

[6] F. Verstraete and J. I. Cirac, Renormalization algorithms for quantum-many body systems in two and higher dimensions, arXiv:cond-mat/0407066.

[7] G. Vidal, Class of Quantum Many-Body States That Can Be Efficiently Simulated, Phys. Rev. Lett. 101, 110501 (2008).

[8] L. Vanderstraeten, J. Haegeman, and F. Verstraete, Tangentspace methods for uniform matrix product states, SciPost Phys. Lect. Notes 7 (2019), doi: 10.21468/SciPostPhysLectNotes.7.
[9] S. Paeckel, T. Köhler, A. Swoboda, S. R. Manmana, U. Schollwöck, and C. Hubig, Time-evolution methods for matrixproduct states, Ann. Phys. 411, 167998 (2019).

[10] E. M. Stoudenmire and S. R. White, Sliced Basis Density Matrix Renormalization Group for Electronic Structure, Phys. Rev. Lett. 119, 046401 (2017).

[11] M. Motta, C. Genovese, F. Ma, Z.-H. Cui, R. Sawaya, G. K.-L. Chan, N. Chepiga, P. Helms, C. Jiménez-Hoyos, A. J. Millis, U. Ray, E. Ronca, H. Shi, S. Sorella, E. M. Stoudenmire, S. R. White, and S. Zhang (Simons Collaboration on the Many-Electron Problem), Ground-State Properties of the Hydrogen Chain: Dimerization, Insulator-to-Metal Transition, and Magnetic Phases, Phys. Rev. X 10, 031058 (2020).

[12] J. Haegeman, S. Michalakis, B. Nachtergaele, T. J. Osborne, N. Schuch, and F. Verstraete, Elementary Excitations in Gapped Quantum Spin Systems, Phys. Rev. Lett. 111, 080401 (2013).

[13] M. Mena, N. Hänni, S. Ward, E. Hirtenlechner, R. Bewley, C. Hubig, U. Schollwöck, B. Normand, K. W. Krämer, D. F. McMorrow, and C. Rüegg, Thermal Control of Spin Excitations 
in the Coupled Ising-Chain Material $\mathrm{RbCoCl}_{3}$, Phys. Rev. Lett. 124, 257201 (2020).

[14] B. Pirvu, J. Haegeman, and F. Verstraete, Matrix product state based algorithm for determining dispersion relations of quantum spin chains with periodic boundary conditions, Phys. Rev. B 85, 035130 (2012).

[15] L. Vanderstraeten, J. Haegeman, and F. Verstraete, Simulating excitation spectra with projected entangled-pair states, Phys. Rev. B 99, 165121 (2019).

[16] B. Ponsioen and P. Corboz, Excitations with projected entangled pair states using the corner transfer matrix method, Phys. Rev. B 101, 195109 (2020).

[17] L. Vanderstraeten, M. Mariën, F. Verstraete, and J. Haegeman, Excitations and the tangent space of projected entangled-pair states, Phys. Rev. B 92, 201111(R) (2015).

[18] P. Corboz, Variational optimization with infinite projected entangled-pair states, Phys. Rev. B 94, 035133 (2016).

[19] I. P. McCulloch, From density-matrix renormalization group to matrix product states, J. Stat. Mech.: Theory Exp. (2007) P10014.

[20] L. Michel and I. P. McCulloch, Schur forms of matrix product operators in the infinite limit, arXiv:1008.4667.

[21] M. E. Peskin and D. V. Schroeder, An Introduction to Quantum Field Theory (Addison-Wesley, Reading, 1995).

[22] A. Altland and B. D. Simons, Condensed Matter Field Theory, 2nd ed. (Cambridge University Press, Cambridge, 2010).

[23] J. Haegeman and F. Verstraete, Diagonalizing transfer matrices and matrix product operators: A medley of exact and computational methods, Annu. Rev. Condens. Matter Phys. 8, 355 (2017).

[24] M. Bartholomew-Biggs, S. Brown, B. Christianson, and L. Dixon, Automatic differentiation of algorithms, J. Comput. Appl. Math. 124, 171 (2000).

[25] A. G. Baydin, B. A. Pearlmutter, A. A. Radul, and J. M. Siskind, Automatic differentiation in machine learning: A survey, J. Mach. Learn. Res. 18, 1 (2018).

[26] H.-J. Liao, J.-G. Liu, L. Wang, and T. Xiang, Differentiable Programming Tensor Networks, Phys. Rev. X 9, 031041 (2019).

[27] The variational uniform MPS algorithm for infinite-size systems [52] can provide a good initial tensor.

[28] A. Auerbach, Interacting Electrons and Quantum Magnetism, Graduate Texts in Contemporary Physics (Springer, Berlin, 1994).

[29] J. Haegeman, B. Pirvu, D. J. Weir, J. I. Cirac, T. J. Osborne, H. Verschelde, and F. Verstraete, Variational matrix product ansatz for dispersion relations, Phys. Rev. B 85, 100408(R) (2012).

[30] Y. Zou, A. Milsted, and G. Vidal, Conformal Data and Renormalization Group Flow in Critical Quantum Spin Chains Using Periodic Uniform Matrix Product States, Phys. Rev. Lett. 121, 230402 (2018).

[31] L. Vanderstraeten, M. Van Damme, H. P. Büchler, and F. Verstraete, Quasiparticles in Quantum Spin Chains with LongRange Interactions, Phys. Rev. Lett. 121, 090603 (2018).

[32] Due to the gauge degree of freedom and orthogonality to the ground state, in each momentum sector, one can obtain $(d-1) D^{2}$ valid solutions for excited states [29].

[33] J. Haegeman, M. Mariën, T. J. Osborne, and F. Verstraete, Geometry of matrix product states: Metric, parallel transport, and curvature, J. Math. Phys. 55, 021902 (2014).

[34] A. Paszke, S. Gross, S. Chintala, G. Chanan, E. Yang, Z. DeVito, Z. Lin, A. Desmaison, L. Antiga, and A. Lerer, Automatic differentiation in PyTorch, in Proceedings of the 31st Conference on Neural Information Processing Systems (NIPS 2017), Long Beach, CA, USA (Curran Associates, Red Hook, NY, 2017).

[35] S. Szalay, M. Pfeffer, V. Murg, G. Barcza, F. Verstraete, R. Schneider, and Ö. Legeza, Tensor product methods and entanglement optimization for ab initio quantum chemistry, Int. J. Quantum Chem. 115, 1342 (2015).

[36] B. Pirvu, V. Murg, J. I. Cirac, and F. Verstraete, Matrix product operator representations, New J. Phys. 12, 025012 (2010).

[37] M. J. O'Rourke, Z. Li, and G. K.-L. Chan, Efficient representation of long-range interactions in tensor network algorithms, Phys. Rev. B 98, 205127 (2018).

[38] Z. Li, M. J. O'Rourke, and G. K.-L. Chan, Generalization of the exponential basis for tensor network representations of longrange interactions in two and three dimensions, Phys. Rev. B 100, 155121 (2019).

[39] M. J. O'Rourke and G. K.-L. Chan, Simplified and improved approach to tensor network operators in two dimensions, Phys. Rev. B 101, 205142 (2020).

[40] We have numerically verified this with the transverse field Ising model.

[41] C. G. West, A. Garcia-Saez, and T.-C. Wei, Efficient evaluation of high-order moments and cumulants in tensor network states, Phys. Rev. B 92, 115103 (2015).

[42] I. Affleck and R. A. Weston, Theory of near-zero-wave-vector neutron scattering in Haldane-gap antiferromagnets, Phys. Rev. B 45, 4667 (1992).

[43] S. R. White and I. Affleck, Spectral function for the $s=1$ Heisenberg antiferromagetic chain, Phys. Rev. B 77, 134437 (2008).

[44] F. Ferrari and F. Becca, Dynamical Structure Factor of the $J_{1}-J_{2}$ Heisenberg Model on the Triangular Lattice: Magnons, Spinons, and Gauge Fields, Phys. Rev. X 9, 031026 (2019).

[45] F. C. Alcaraz, M. I. Berganza, and G. Sierra, Entanglement of Low-Energy Excitations in Conformal Field Theory, Phys. Rev. Lett. 106, 201601 (2011).

[46] J. Mölter, T. Barthel, U. Schollwöck, and V. Alba, Bound states and entanglement in the excited states of quantum spin chains, J. Stat. Mech.: Theory Exp. (2014) P10029.

[47] O. A. Castro-Alvaredo, C. De Fazio, B. Doyon, and I. M. Szécsényi, Entanglement Content of Quasiparticle Excitations, Phys. Rev. Lett. 121, 170602 (2018).

[48] J. Zhang and M. A. Rajabpour, Universal Rényi entropy of quasiparticle excitations, arXiv:2010.13973.

[49] J. Eisert, M. Cramer, and M. B. Plenio, Colloquium: Area laws for the entanglement entropy, Rev. Mod. Phys. 82, 277 (2010).

[50] M. Van Damme, R. Vanhove, J. Haegeman, F. Verstraete, and L. Vanderstraeten, Efficient MPS methods for extracting spectral information on rings and cylinders, arXiv:2102.10982.

[51] B. Ponsioen and P. Corboz (unpublished).

[52] V. Zauner-Stauber, L. Vanderstraeten, M. T. Fishman, F. Verstraete, and J. Haegeman, Variational optimization algorithms for uniform matrix product states, Phys. Rev. B 97, 045145 (2018). 